\title{
ARDUINO application prototype as an Oximeter
}

\author{
Joel Flores ${ }^{1}$, Juanita Nancy Mondragón ${ }^{2}$, Brian Armando Peña ${ }^{3}$
}

Professor, Communications and Electronic Engineering Department (ICE), ESIME-ZAC, IPN, Mexico City, Mexico ${ }^{1-2}$

Assistant Professor, Communications and Electronic Engineering Department (ICE), ESIME-ZAC, IPN,

Mexico City, Mexico ${ }^{3}$

\begin{abstract}
The present work proposes a prototype of the ARDUINO UNO application as an oximeter, with components that are obtained in the national market and with low power consumption, as well as being portable, economical and easy to use; and with this it can be built to make it affordable in homes to monitor the detection of oxygenation levels present in the blood in health emergencies.
\end{abstract}

Keywords: ARDUINO, oximeter, blood oxygen saturation, reductive hemoglobin, oxyhemoglobin, optical sensors.

\section{INTRODUCTION}

Pulse oximetry is the measurement of oxygen transported by hemoglobin, it evaluates oxygen saturation by expressing the portion of oxygen that mixes with hemoglobin in chemical terms, to form oxyhemoglobin, a molecule in charge of transporting oxygen to tissues. When measuring oxygen saturation, the amount of oxygen that mixes with hemoglobin is being quantified, so it is a relative and not an absolute measure, so it does not indicate the amount of oxygen in the blood that reaches the tissues, more either indicates the relationship between the amount of hemoglobin and the amount of oxygenated hemoglobin (oxyhemoglobin) [1].

Through non-invasive continuous monitoring, the oximeter expresses changes in a person's oxygenation levels and indicates if there are heart problems based on the changes in the levels. In this way, it helps to prevent possible congenital heart disease early.

Normal saturation levels in the human body guarantee an adequate amount of oxygen to the cells of the human body, there is an ideal percentage of oxygen in the blood, a range of $95 \%$ to $100 \%[2,3,4]$. When there is a percentage below $95 \%$, there are problems with the saturation level, which tends to have congenital heart defects.

\section{DESCRIPTION}

The prototype design consists of five design stages: emission-reception (optical elements), amplification, development (microcontroller), visualization and energy (power supply), as shown in Fig. 1.

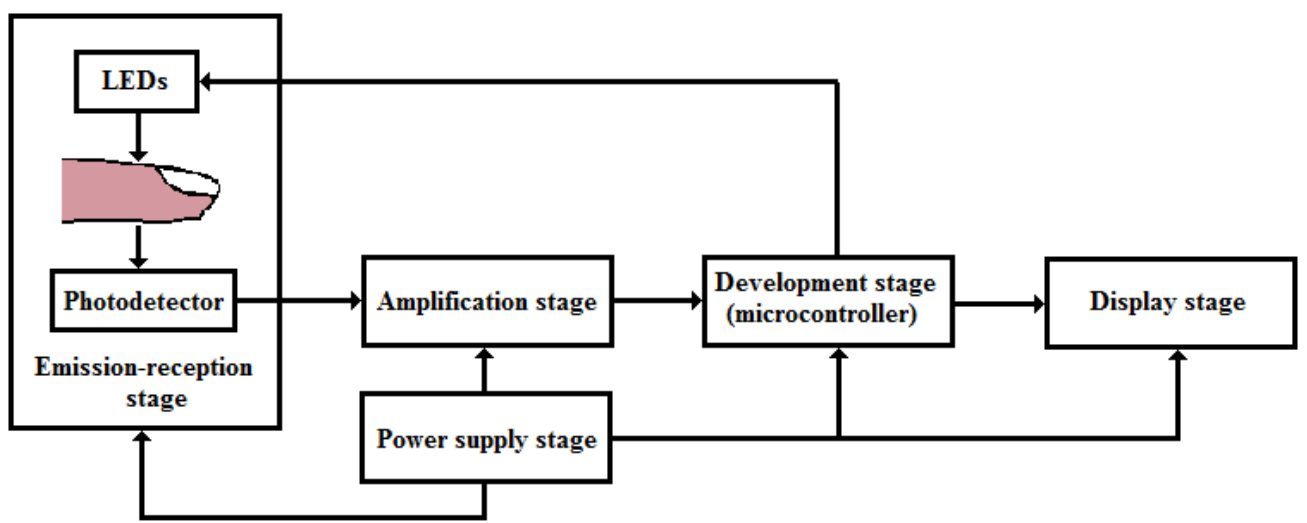

Fig. 1 Block diagram of the prototype design proposal

The emission-reception stage is defined by two optical sensors, for the emission process two light sources (LED) are used at different wavelengths, LED 1 to emit red light (the wavelength is $660 \mathrm{~nm}$ ) and the 2 IR LED emits an infrared wavelength (the wavelength is $880 \mathrm{~nm}$ ), these emission sources are connected to a $5 \mathrm{~V}$ direct current power supply, 


\section{DOI 10.17148/IJIREEICE.2020.81201}

through a resistor Rlim_X to limit the passage current $(20 \mathrm{~mA})$ and avoid damaging the device. The emitters send light at different wavelengths, towards the phototransistors (receivers) so that the light hits the pn junction, thus generating current variations (data / information), in the middle of these (emitters-receivers) the finger is placed of the person, this causes that there are variations of current intensities due to the different absorptions of both red and infrared light, all these variations are captured by the phototransistors and taken as information. The electrical diagram of the emissionreception stage is shown in Fig. 2.

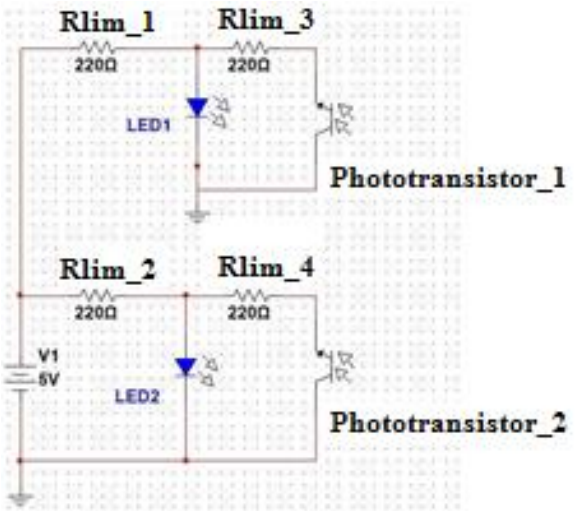

Fig. 2 Electrical diagram of the emission-reception stage

A mold is designed with silicone in the shape of a clamp, in said mold the LEDs (red and infrared light beam) and the phototransistors are mounted inside it and in turn they are connected by cables (the receivers are welded on the upper part and the bottom emitters). It is precisely in this mold that the two beams of different wavelengths of lights can be concentrated on the tip of the human nail. In Fig. 3 the clamp is shown where people's finger is inserted.

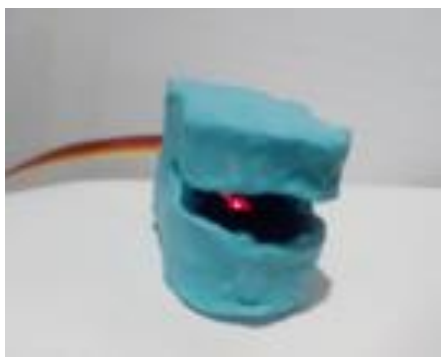

Fig. 3 Mold of the clamp for positioning the fingers

An amplification stage is required, since the data obtained from the photosensitive elements is very small data or small signals, so a gain of 100 times is proposed to handle the data with an adequate amplitude required on the development board. For this design the ML358P operational amplifier configured as non-inverting is used [6]. In Fig. 4 shows the complete electrical diagram, in which the two LEDs are joined with the Receivers that are two phototransistors and the amplifiers. A magnitude 5 V DC power supply is used to power the stages. It is also shown that each amplifier output is connected to a peripheral on the development board, they are labeled Output1_ARDUINO and Output2_ARDUINO.

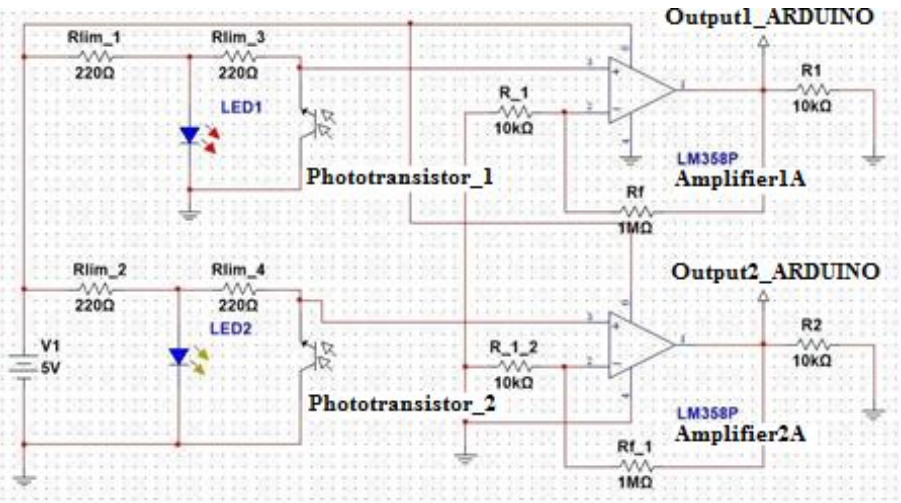

Fig. 4 Electrical diagram designed for the measurement system 
International Journal of Innovative Research in Electrical, Electronics, Instrumentation and Control Engineering

Vol. 8, Issue 12, December 2020

\section{DOI 10.17148/IJIREEICE.2020.81201}

In the development stage, the ARDUINO UNO microcontroller is used, this is a card that is connected to a computer to program it, this device is proposed, or better called development board, due to its technical specifications, its free code, its easy handling, the variety of applications that can be made and its low cost [5].This card needs to be programmed and energized with a direct current power supply of magnitude $5 \mathrm{~V}$. It is precisely at this stage where the secret of source code programming is found. The source code is shown in Fig. 5.

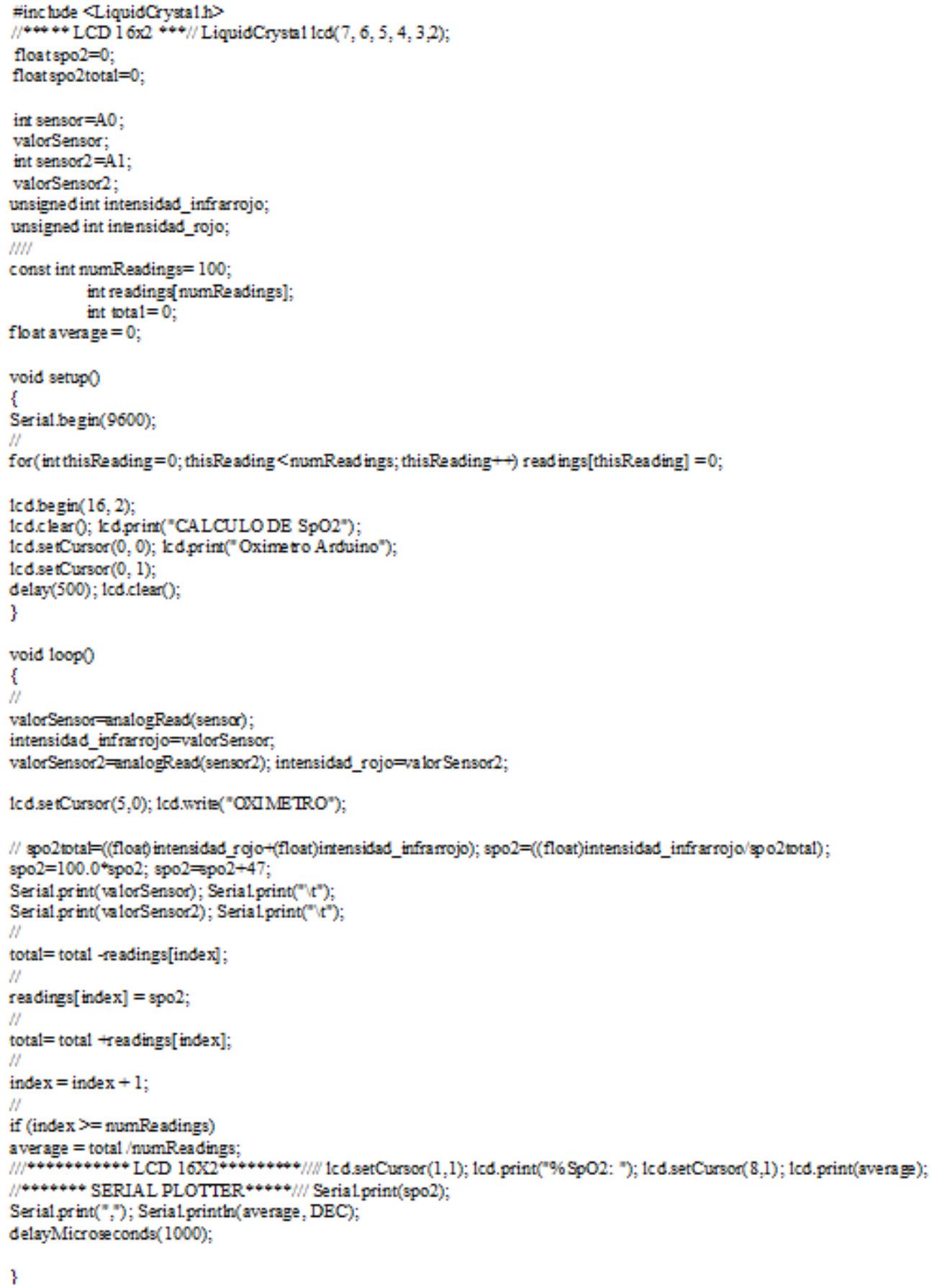

Fig. 5 Source code

In the display stage, a 16x2 liquid crystal display (LCD) is used to display the information, this device is powered by a $5 \mathrm{~V}$ direct current power supply.

According to the LiquidCrystal library that is incorporated in the ARDUINO IDE installation, it allows to control the screen easily, whereby pins 5, 4, 3, and 2 are connected to the DB4-DB7 pins of the LCD screen. Similarly, pins 12 and 


\section{DOI 10.17148/IJIREEICE.2020.81201}

11 are connected to the mode selector (Read/Write) and to enable the screen to receive information, respectively. On the other hand, the inputs of the ARDUINO A0 and A1 board are connected to the output of the two amplifiers, these outputs are responsible for sending the information. In other words, all the outputs of the development board are connected to the LCD screen and to the outputs of the amplifiers, in the same way it happens with the power supply.

In Fig. 6 the finished prototype is shown, as a summary the final electrical diagram is assembled on the test board, its input signals, which are the receivers, are connected by a cable to the clamp mold that contains the emitters.

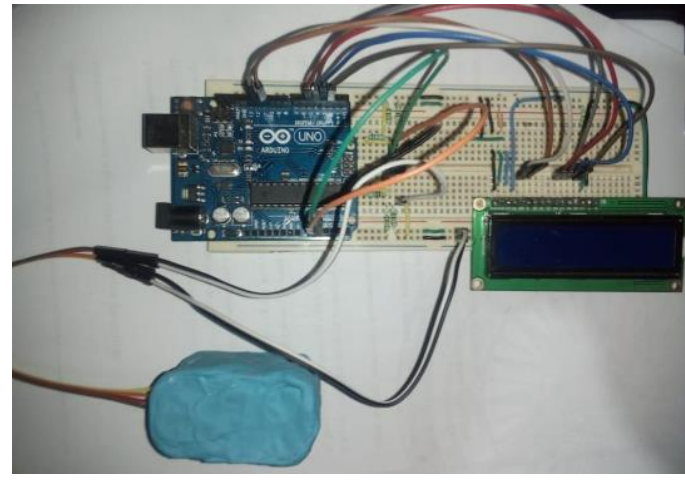

Fig. 6 Final prototype of the oximeter

\section{RESULTS}

The oxygen saturation present in the blood requires the processing of two signals, a signal from the phototransistor that receives the wavelength of red light, this signal is interpreted as absorption of hemoglobin $(\mathrm{Hb})$ and in the same way it requires another signal to process, which is the one from the phototransistor that receives the infrared wavelength. This is the signal that is interpreted as absorption of oxyhemoglobin $\left(\mathrm{HbO}_{2}\right)$.

The ARDUINO IDE application is used to visualize both signals, by programming a source code, in the application the signals of the two different wavelengths can be displayed in a graph through the serial plotter function incorporated in said application. In Fig. 7 the two signals of the different wavelengths are shown, in the blue signal the infrared wavelength $\left(I_{I R}\right)$ is observed and in the red signal $\left(I_{R}\right)$ the wavelength of the red light. Which is obtained from the different signals of the phototransistors, showing the signal of oxyhemoglobin and hemoglobin separately.

According to Lambert Beer's law, the average oxygen saturation in the blood is calculated (adding the red and infrared light signal), as shown in equation 1 and 2.

$$
\begin{array}{ll}
\mathrm{SpO}_{2 T}=I_{R}+I_{I R} & \text { Equation (1) Sum of both signals } \\
\% \mathrm{SpO}_{2}=\frac{I_{R}}{\mathrm{SpO}_{2 T}} \times 100 & \text { Equation (2) Average oxygen saturation }
\end{array}
$$

Where:

$\mathrm{SpO}_{2} \mathrm{~T}$ is variable from the total of the red and infrared signals [Dimensionless]

$\mathrm{I}_{\mathrm{R}}$ is the red intensity [Dimensionless]

$\mathrm{I}_{\mathrm{IR}}$ is the infrared intensity [Dimensionless]

$\% \mathrm{SpO}_{2}$ is the oxygenation saturation percentage [Dimensionless]

In the same Fig. 7 the green signal of the sum of the red and infrared light signals is shown, in the same way the signal of the percentage of oxygen saturation levels present in orange blood is shown.

As can be seen, the orange signal is the one that is used to show the oxygen saturation present in the blood, which has a margin of error of $4 \%$ (it shows values below the normal ranges of saturation present in the blood ) this error is a very important factor to consider when calibrating the prototype.

The IEC 60601-1 (1988-12) standard establishes standards for the safety of all medical electrical equipment used by or under the supervision of qualified personnel in the medical environment. It also contains certain requirements for reliable operation to ensure safety. For the case of reliable operation of the oximeter it is with an error rate of $2 \%$. 
Vol. 8, Issue 12, December 2020

DOI 10.17148/IJIREEICE.2020.81201

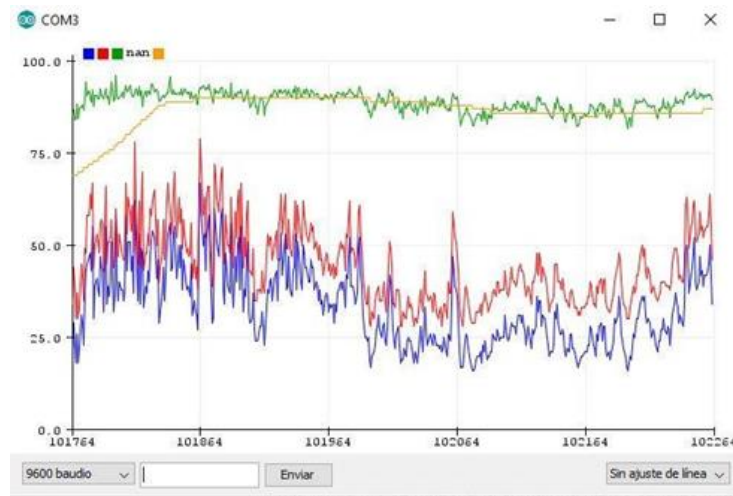

Fig. 7 Red, infrared and average oxygen saturation signals

\section{CONCLUSION}

When developing the prototype, it requires specific devices so that the signals obtained do not have too much noise, otherwise the oxygen saturation will have an error and will not be reliable for interpretation. Therefore, it is important that this type of device use phototransistors for medical use that are specially designed to measure the red spectrum, which are used for the manufacture of oximeters. After doing the simulations and checking the reliability of the prototype, it was precisely discovered that for any design to measure oxygen saturation it is essential to have a phototransistor for medical use, since when using another type of phototransistor, noisy signals are obtained.

The mold of the clamp that was built was handmade, which said mold could be more detailed and little more compact, if a $3 \mathrm{D}$ printer is used. It is considered that the prototype meets the main objective of this work, when designing a measurement system for oxygen saturation levels, unfortunately the advance only shows the percentage of oxygen saturation in graphical form, which would depend on a PC. The next step is to display a reading on the screen.

Finally, the development of an oximeter or monitoring system, currently under the circumstances of the pandemic caused by the COVID-19 virus, is essential since a main cause of this virus is the lack of oxygen in the human body, this lack of oxygen makes people enter a hospital in a very serious state of health, this for not realizing that their body was entering a low oxygen saturation constant. So it is necessary to have this type of monitoring instruments in our homes.

\section{REFERENCES}

[1] R.P. Garrido, M. González, M. García and I. Expósito, "Ergospirometric desaturation patterns as a function of age", International Journal of Medicine and Sciences of Physical Activity and Sport., vol. 5, pp. 100-117, Nov. 2005.

[2] P. J. F. Restrepo and E. C. Rodríguez, Blood gases, physiology of breathing and acute respiratory failure. 7th ed., Ed. Médica Panamericana S.A, Bogotá, Colombia, 2005.

[3] D. M. Abedrapo and J. P. Garces, Physiology of the blood, Ed. Santiago, Chile: Pontificia Universidad Católica de Chile, 2003.

[4] J. P. Amigo. (2019) Normal pulsation levels. [Online]. Available: https://revistadigital.inesem.es/biosanitario/pulsaciones/ \#:\%7E:text=Ni\%C3\%B1os\%20de\%205\%20a\%206,a\%2060\%20latidos\%20por\%20minuto.

[5] (2019) Arduino Official Store website [Online]. Available: https://store.arduino.cc/arduino-uno-rev3

[6] R. F. Couglin, and F. F. Driscoll, Operational amplifiers and linear integrated circuits, Ed. NJ, Estados Unidos: Prentice Hall, 1994.

\section{BIOGRAPHY}

Joel Flores Martínez received his B.Sc. in electronics and communications engineering from ESIME Zacatenco IPN, Mexico, in 1997 and M.Sc. degree in Bioelectronics from CINVESTAV IPN, Mexico in 2000. Currently he is a professor at the Department of Electronics and Communications engineering in ESIME Zacatenco. His research interests are in acoustics and biomedical engineering.

Juanita Nancy Mondragón Medina received her B.Sc. in electronics and communications engineering from ESIME Zacatenco IPN, Mexico, in 2005. Currently she's a teacher at the Department of Electronics and Communications engineering in ESIME Zacatenco. Her research interests are in electrical communications.

Brian Armando Peña González received his B.Sc. in electronics and communications engineering from ESIME Zacatenco IPN, Mexico, in 2019. Currently he's an assistant professor at the Department of Electronics and Communications engineering in ESIME Zacatenco. His research interests are in electrical communications. 